\title{
Un enfoque estratégico de la motivación laboral y la satisfacción laboral
}

\author{
LORENZO REVUELTO-TABOADA ${ }^{a}$
}

pp. 7-26
RESUMEN La literatura especializada nos ha mostrado durante décadas la importancia capital que tiene para las organizaciones contar con unos trabajadores motivados y suficientemente satisfechos o, cuanto menos, no insatisfechos con su trabajo. Esto exige, por una parte, adoptar una perspectiva estratégica que implica dinamismo, anticipación y capacidad para adaptarse a los cambios en el contexto organizativo y en las personas, sus valores, sus necesidades y sus prioridades. Es necesario que las organizaciones se replanteen de forma continua qué pueden hacer para atraer, retener y motivar a las personas que necesitan (mientras las necesitan), a fin de lograr sus objetivos en un contexto dinámico. Por otra parte, dadas las evidentes interrelaciones que existen entre las distintas políticas y prácticas de recursos humanos, es necesario adoptar un planteamiento integral y sistemático en lo que se refiere a la dirección de recursos humanos, en general, y a la motivación en particular. El presente trabajo comienza con el repaso de algunos datos reveladores sobre el deterioro de la relación empleados-organización, así como por analizar el contexto de esta relación al poner el énfasis en algunas de las posibles causas de este deterioro. Luego, analiza la motivación como función y proceso para abordar, a continuación, algunas posibilidades interesantes de intervención, desde una perspectiva sistémica y estratégica.

PALABRAS CLAVE enfoque contextual, motivación, perspectiva estratégica, recursos humanos, satisfacción laboral, teoría de las expectativas.

\section{HISTORIA DEL ARTÍCULO}

¿CÓMO CITAR?:

Revuelto-Taboada, L. (2018). Un enfoque estratégico de la motivación laboral y la satisfacción laboral. Perspectiva Empresarial, 5(2), 7-26. http://dx.doi. org/10.16967/rpe.v5n2a1

RECIBIDO: 12 de marzo de 2018 APROBADO: 15 de agosto de 2018

CORRESPONDENCIA:

Lorenzo Revuelto-Taboada. Universidad de Valencia, Departamento de Dirección de Empresas “Juan José Renau Piqueras”, Avda. Los Naranjos, Edificio Departamental Oriental, 46022, Valencia (España). 


\section{¿CóMO CITO EL ARTíCULO? HOW TO CITE THIS PAPER?}

CHICAGO:

Revuelto-Taboada, Lorenzo. 2018. "Un enfoque estratégico de la motivación laboral y la satisfacción laboral". Perspectiva Empresarial 5(2): 7-26. http://dx.doi. org/10.16967/rpe.v5n2a1

MLA:

Revuelto-Taboada, Lorenzo. "Un enfoque estratégico de la motivación laboral y la satisfacción laboral". Perspectiva Empresarial 5.2 (2018): 7-26. Digital. http:// dx.doi.org/10.16967/rpe. v5n2a1

\section{A Strategic Approach to Work Motivation and Job Satisfaction}

ABSTRACT Specialized literature has shown us for decades the paramount importance for organizations to have workers that are motivated and sufficiently satisfied or, at least, not dissatisfied with their job. This requires, on the one hand, adopting a strategic perspective, which implies dynamism, anticipation and ability to adapt to changes both in the organizational context and in individuals, their values, needs and priorities. Organizations need to continually rethink what they can do to attract, retain and motivate the people they need (while they need them) to achieve their objectives in a dynamic context. On the other hand, given the obvious interrelationships that exist between the different human resource policies and practices, it is necessary to adopt a comprehensive and systematic approach in terms of human resource management in general and motivation in particular. This work begins by reviewing some revealing data about the deterioration of the employee-organization relationship, analyzing its context and emphasizing some of the possible reasons for this deterioration. Subsequently, motivation as a function and process is analyzed to later address some interesting possibilities of intervention, from a systemic and strategic perspective.

KEYWORDS contextual approach, motivation, strategic perspective, human resources, job satisfaction, expectations theory.

\section{Um enfoque estratégico da motivação laboral e da satisfação laboral}

RESUMO A literatura especializada tem nos mostrado, durante décadas, a importância capital que contar com trabalhadores motivados e suficientemente satisfeitos ou, pelo menos, não insatisfeitos com o seu trabalho representa para as organizações. Isso exige, por um lado, adotar uma perspectiva estratégica, que implica dinamismo, antecipação e capacidade para adaptar-se às mudanças no contexto organizativo e nas pessoas, seus valores, suas necessidades e suas prioridades. É necessário que as organizações repensem, continuamente, o que podem fazer para atrair, reter e motivar as pessoas que precisam (enquanto precisarem delas), para conseguir seus objetivos em um contexto dinâmico. Por outro lado, dadas as evidentes inter-relações que existem entre as diferentes políticas e práticas de recursos humanos. É necessário adotar um planejamento integral e sistemático no que diz respeito à direção de recursos humanos, em geral, e à motivação em particular. 0 presente trabalho começa repassando alguns dados reveladores sobre o desgaste da relação empregado-organização, analisar o contexto da mesma e dar ênfase em algumas das possíveis causas desse desgaste. Posteriormente, analisa a motivação como função e processo para abordar, depois, algumas possibilidades interessantes de intervenção, a partir de uma perspectiva sistêmica e estratégica..

PALAVRAS CHAVE enfoque contextual; motivação; perspectiva estratégica; recursos humanos; satisfação laboral; teoria das expectativas. 


\section{Introducción}

El estudio de la motivación y la satisfacción laboral en la empresa no constituye, en absoluto, un tema novedoso, pues son innumerables las investigaciones realizadas desde mediados del siglo xx en torno a estos conceptos. Estas investigaciones han logrado amplios consensos, por ejemplo, con respecto a las relaciones negativas entre la satisfacción laboral y la rotación laboral (Akerlof, Rose y Yellen, 1988; Bluedorn, 1982; Hom, Caranikas-Walker, Prussia y Griffeth, 1992; Lee, 1988; McEvoy y Cascio, 1985); la predisposición a abandonar la empresa, lo cual predice el subsiguiente abandono de esta (Blau, 1993; Lum, Kervin, Clark, Reid y Sirola, 1998; Shore, Newton y Thorton, 1990; Tett y Meyer, 1993); y los niveles de absentismo (Clegg, 1983; Dalton y Mesch, 1991; Mangione y Quinn, 1975). Lo anterior nos permite afirmar que la insatisfacción conlleva costes importantes en reclutamiento y selección, formación, ineficiencias en la curva de aprendizaje, pérdidas en productividad, pérdida de clientes, etc. (Brown y Mitchell, 1993; Cascio, 1991; Tziner y Birati, 1996).

También se han encontrado correlaciones significativas entre satisfacción y ratios de Burn-out (Bacharach, Bamberger y Conley, 1991; Shirom, 1989); salud psicológica (Jex y Gudanowsky, 1992; Spector, Dwyer y Jex, 1988); y salud física (Begley y Czajka, 1993; Lee, Ashford y Bobko, 1990) de los empleados. Asimismo, se han encontrado relaciones positivas entre satisfacción laboral y compromiso organizativo (Mathieu, 1991; Mathieu y Zajac, 1990); la predisposición a cooperar con otros, operativizada mediante el constructo de ciudadanía organizativa (Bateman y Organ, 1983; Farh, Podsakoff y Organ, 1990; McNeely y Meglino, 1994; Motowidlo, 1984; Organ y Ryan, 1995); y la calidad de los servicios medida en términos de satisfacción del consumidor (Fosam, Grimsley y Wisher, 1998; Rafaeli, 1989; Rogers, Clow y Kash, 1994).

En definitiva, no puede quedar duda de la importancia capital que puede tener para la organización contar con unos trabajadores motivados y suficientemente satisfechos o, cuanto menos, no insatisfechos con su trabajo. Cabe destacar, además, que las sociedades cambian, las empresas cambian y también lo hacen las personas, sus valores, sus necesidades y sus prioridades, lo cual obliga a replantearse de forma continua qué pueden hacer las empresas para atraer, retener y motivar a las personas que necesitan (mientras las necesitan), a fin de adaptarse a su contexto organizativo y alcanzar sus objetivos. Por tanto, el estudio de la motivación y la satisfacción laboral constituye un tema de actualidad, y nos atrevemos a predecir que seguirá siéndolo en las décadas venideras. Sin embargo, ¿qué hay de realmente novedoso en la situación actual que parece exigir una revisión de las políticas y prácticas de motivación en las empresas? En nuestra opinión, existen diferentes cuestiones a tener en cuenta, las cuales se exponen a continuación.

En primer lugar, consideramos necesario llamar la atención sobre la cantidad de datos objetivos que parecen revelar niveles crecientes de absentismo, de predisposición a abandonar las empresas, de abandono efectivo y un compromiso decreciente de los empleados. Es un fenómeno estudiado, sobre todo, en economías más desarrolladas, pero que parece afectar cada vez más a un mayor número de países. Por citar algunos datos ilustrativos, de entre los muchos que podrían ofrecerse, se presentan los siguientes:

- Los datos de la encuesta de Korn Ferry International (2003) revelan que el $62 \%$ de los más de 2500 ejecutivos analizados, pertenecientes a distintos países a lo largo y ancho del planeta, estaban descontentos en sus empleos actuales.

- Pollster Gallup señala que el 80\% de los trabajadores británicos muestran un bajo compromiso con su trabajo, y el $25 \%$ de estos se encuentran "activamente desconectados" de sus empleadores, de modo que incurren con frecuencia en sabotajes. La situación es aún peor en Francia, donde solo el 12\% de los empleados se sienten comprometidos con su trabajo. Los datos revelan que no es un problema exclusivamente de Occidente ya que, por ejemplo, en Singapur el 17\% de los empleados se hallan también activamente desconectados, de manera que generan una fuerza corrosiva para la organización (Deloitte Research, 2004).

- La encuesta Conference Board (2005), realizada en 5000 hogares en Estados Unidos, reveló -entre otras cosas- que el $67 \%$ de los empleados no se identificaban con los objetivos de sus empleadores, casi el $50 \%$ se sentían desconectados de estos y al $25 \%$ solo les preocupaba cobrar su cheque. 
- Frauenheim (2006), con base en datos de Metlife, señala que el $22 \%$ de los empleados encuestados habían cambiado de puesto de trabajo en los 18 meses anteriores.

- Según Capelli (2006), la antigüedad media del personal masculino (también ejecutivos senior) había descendido ampliamente entre 1983 y el 2000. Estudios más recientes parecen confirmar esta tendencia.

- La encuesta Regus Business Tracker (2010) arrojó que el 53\% de los trabajadores (de un universo de 10000 pertenecientes a diversos países) estaría dispuesto a abandonar su puesto actual por falta de oportunidades de promoción, o porque no se tuvieran en cuenta los resultados. Otros factores importantes para justificar un posible abandono serían la falta de visión estratégica, deficiencias de comunicación de los directivos o estrés y sobrecarga de trabajo.

- La Metlife Foundation (2012), en el estudio sobre tendencias de los beneficios para empleados, incluye tres datos relevantes: a) una de cada tres personas espera cambiar de empresa, b) por séptimo año consecutivo disminuye la lealtad de los empleados, c) casi el $50 \%$ aspira a que la empresa les siga ayudando con beneficios sociales.

- Según datos que arroja el Índice de Felicidad Organizacional de Ibeoramérica, elaborado por Universia en el 2016 en Argentina, Colombia, Chile, México, Perú y España, la mayoría de los 7571 empleados encuestados están descontentos con su situación laboral y son proclives a cambiar de trabajo. Los colombianos se muestran a la cabeza de los empleados felices, eso sí, con un 42\%, mientras que, en el otro extremo, encontramos el caso español, en el que el $78 \%$ de los trabajadores se muestra descontento con su trabajo.

Por otra parte, cabe destacar el hecho de que mientras el nivel de compromiso de los trabajadores con su empresa parece decrecer de manera peligrosa, aumenta el compromiso con la profesión, con la propia carrera profesional planteada cada vez más como una sucesión natural de empleos y empresas (Fernández-Mateo, 2002). Conseguir prestigio dentro de la profesión pasa a constituir la clave para incrementar el salario y participar en proyectos atractivos, de manera que muestra el empleado una fuerte predisposición a cambiar de empresa siempre que esto favorezca este progreso profesional.

Todos estos datos, preocupantes de por sí, lo parecen aún más si nos atenemos a lo que la literatura sobre dirección estratégica de recursos humanos (RRHH) señala en relación con el papel de las personas en la empresa. Con base, fundamentalmente, en argumentos de la teoría de recursos y capacidades (Barney y Wright, 1998; Boxall, 1996; Kamoche, 1996; Lado y Wilson, 1994; Wright, Dunford y Snell, 2001), y, en menor medida, de la teoría del capital humano (Cascio, W., 1991; Flamholtz y Lacey, 1981; Lepak y Snell, 1999; Snell y Dean, 1992), la academia destaca el papel estratégico de los RRHH como fuente de ventaja competitiva.

Una multitud de expertos en la materia, tanto en el ámbito profesional como en el científico, destacan la importancia de atraer, retener y aprovechar el talento de los empleados para el éxito de las organizaciones. Al respecto existe una amplia literatura sobre prácticas o sistemas de prácticas de alto rendimiento o compromiso, la cual destaca las bondades de ciertas prácticas de RRHH y sus efectos sobre los resultados. Entre estos trabajos podemos destacar, entre otros, los de autores como Huselid (1995), MacDuffie (1995), Becker y Gerhart (1996), Delaney y Huselid (1996), Ichniowski, Shaw y Prennushi (1997), Becker y Huselid (1998), Pfeffer (1998), Guthrie (2001), y Way (2002).

Una vez se ha resaltado la dimensión del problema a abordar y las posibilidades de intervención, en el siguiente apartado se aborda el análisis del contexto actual de la motivación y las posibles causas del deterioro de la relación empleado-organización. A continuación, se revisa lo que hemos aprendido del estudio de la motivación en cuanto función y como proceso, para luego pasar a analizar las posibilidades de intervención y tomar como referencia las necesidades y las preferencias de los empleados. Finalmente, se razona la necesidad de adoptar una perspectiva sistémica y estratégica de la motivación que plantee soluciones integrales al tener en cuenta las interrelaciones entre las diferentes políticas y prácticas de RRHH. Finalmente, se ofrecen una serie de conclusiones, contribuciones y líneas de investigación futura. 


\section{El contexto actual de la motivación laboral}

Enfrentados a los datos señalados, no faltan aquellos (sobre todo del lado de la empresa) que ponen en juego elementos como, por ejemplo, la crisis de valores, la pérdida de la ética en el trabajo y del trabajo, las crecientes expectativas del trabajador asociadas a los crecientes niveles de desarrollo o, incluso, a las conductas hedonistas que generan las sociedades opulentas, en cuanto factores fundamentales a la hora de explicar esa cada vez más crítica, compleja y potencialmente conflictiva relación del empleado con la empresa.

Si bien no podemos ni debemos descartar dichos factores como elementos importantes, deberíamos plantearnos en qué medida son la causa de los problemas señalados, y en qué medida son la consecuencia natural del contexto en que se desarrolla la relación laboral. Un contexto que se caracteriza por un decreciente compromiso de la empresa con el trabajador, la ruptura del contrato psicológico en su concepción tradicional, la inseguridad laboral, los crecientes niveles de exigencia de productividad y de actualización de competencias, así como las dificultades para conciliar la vida laboral y familiar. Aunque también es un contexto de aparición de nuevos conceptos como los de empleabilidad (Fugate, Kinicki, y Ashforth, 2004; Hillage y Pollard, 1998; Silla, 2007), y flexiguridad (Cazes y Nesporova, 2007; Wilthagen y Tros, 2004), llamados a jugar un papel importante a la hora de reconfigurar el contrato psicológico, pero todavía no asentados en la mayoría de sociedades y empresas.

Conviene en este punto revisar algunas cuestiones fundamentales que autores tan reputados como Pfeffer (2007) señalan como causas de las actitudes negativas hacia y en el trabajo. En primer lugar, cabe destacar las oleadas de reducciones de tamaño y reestructuraciones que han hecho que los empleados de casi todos los niveles de la organización se sientan menos seguros. Hoy en día, estos procesos afectan a un número cada vez mayor de empresas y se han generalizado en gran parte del orbe. Resulta llamativa la cantidad de empresas importantes que diariamente anuncian recortes en sus plantillas y utiliza para esto términos tales como "despidos", "reducciones de plantilla", "recortes de personal", o bien los eufemismos "ajuste", "redimensionamiento", "eliminación de puestos redundantes" o "reingeniería". Si bien, tradicionalmente, estos recortes han afectado sobre todo a los puestos de baja cualificación (en muchos casos como consecuencia de avances tecnológicos y convulsiones en el sector), en la actualidad afectan cada vez más a los puestos técnicos y directivos. Además, ya no se trata casi de manera exclusiva de fenómenos relacionados con situaciones de crisis o de cambio tecnológico, sino que, con frecuencia, las reducciones de plantilla las llevan a cabo empresas que gozan de una buena situación económica (Francis y Pett, 2004).

En no pocos casos estas reducciones de plantilla han estado vinculadas a procesos de concentración a nivel sectorial, muy frecuentes en sectores como el financiero, el de seguros, el de la industria alimentaria o el de la distribución comercial, etc., en los que impera la lógica del volumen. Si bien el impacto de las oleadas de fusiones, adquisiciones $\mathrm{y}$ absorciones que conllevan estos procesos sobre el empleo varía de forma sustancial de un sector a otro - de manera que es, por ejemplo, menor en el sector de la distribución comercial (Organización Internacional del Trabajo-oIT, 2003)—, siempre provocan duplicidades y solapamientos que se saldan con reducciones de plantilla y, en no pocos casos, precarización de las condiciones de trabajo. A modo de ejemplo, la oit (2001) señalaba que los cálculos más conservadores indicaban la desaparición de al menos 130000 empleos en el sector financiero en Europa Occidental, como resultado de las fusiones y la adquisición en la década de los noventa. La situación al respecto no ha hecho más que empeorar en la primera década del siglo XxI, sobre todo desde el inicio de la crisis financiera internacional.

Por otra parte, resulta devastador para la moral y el compromiso de los trabajadores el hecho de que, en la mayoría de los casos, la respuesta inmediata a las crisis - como fue el caso de la que tuvo su inicio a principios del 2008-, consista en la tramitación, cuanto más urgente mejor, de expedientes de regulación de empleo (en definitiva, despidos colectivos basados en razones objetivas o tecnológicas), sin que se planteen - salvo en casos muy excepcionales- otras alternativas. Alternativas que podrían combinar actuaciones como, por ejemplo, congelación salarial o aumento del porcentaje de componente variable, fomento de la movilidad y la polivalencia, erradicación de horas extraordinarias, reparto de trabajo, inversión en formación, bajas incentivadas, años sabáticos, nuevas iniciativas intraemprendedoras, etc. Así, por ejemplo, en el caso español, 180579 trabajadores se vieron afectados por un expediente 
de regulación de empleo (ERE) en el primer trimestre del 2009, frente a los 11375 en el mismo periodo del 2008, lo que representó un aumento relativo del $1487,5 \%$, lo que nos da una idea de la reacción de las empresas frente a la crisis.

Otro fenómeno vinculado al comercio internacional, a la integración económica y a la globalización -con consecuencias dispares en los países de origen y destino-, es el de la deslocalización o relocalización de actividades (Baldwin y Robert-Nicoud, 2000; Krugman y Venables, 1990; Mouhoud, 1989). Este fenómeno, que afecta a un número cada vez mayor de sectores productivos, sobre todo a los de baja intensidad tecnológica, suele implicar, en la mayoría de los casos, relocalización de actividades productivas en países de bajos costes laborales (en los que la inversión extranjera puede tener efectos muy positivos) y cierre de instalaciones; por tanto, despidos y precarización de las condiciones de trabajo en los países de origen.

A fin de hacernos una idea de la importancia del fenómeno cabe señalar que, si comparamos los datos del Banco Mundial de 1970 y del 2017, la inversión extranjera directa en países de ingresos medio y bajo ha crecido de USD 1508,47 a USD 514 614,99 millones (World Bank Group, 2018). Según datos del Bureau of Economic Analysis (2011), entre 1999 y el 2008 las empresas multinacionales de EE. UU. han destruido $1903000 \mathrm{em}$ pleos en el país, mientras que en el mismo periodo han creado 2358000 empleos en el exterior. En Europa, si bien el proceso se ha ralentizado durante la pasada crisis, la pérdida de puestos de trabajo en los países de origen ha sido importante en las últimas décadas, especialmente en la industria, de modo que son las más afectadas la del automóvil y la electrónica. Independientemente de lo saludable que puedan resultar dichas prácticas para la competitividad de las empresas que las practican, difícilmente cabe esperar compromiso por parte de trabajadores que ven peligrar sus empleos en empresas que, en muchos casos, nunca dejaron de ser rentables.

Como señala Pfeffer (2007, p. 117), "muchas empresas han roto de forma implícita o explícita las promesas que hicieron a sus empleados (...) lo que ha incrementado la reticencia de los empleados a creer en lo que la empresa les dice". El autor proporciona como ejemplos de estas conductas en EE. UU., entre otros factores, la reducción significativa del porcentaje de empleados incluidos en planes de jubilación, de empleados con beneficios sanitario tras su jubilación, o de los empleados con cobertura sanitaria subvencionada por su empleador. Lo que viene acompañado, en épocas de crisis, de despidos, reducciones de salario y la consecuente pérdida de poder adquisitivo.

Sin embargo, quizás lo más importante es la percepción de que el contrato psicológico se ha roto, y que las empresas ni pueden ni quieren garantizar empleo a cambio de lealtad, compromiso y productividad. Además, lo que es más grave de cara el futuro es que, al ser comprensible que las empresas no siempre, de hecho, o casi nunca, pueden garantizar el empleo a largo plazo, la mayoría de ellas tampoco se esfuerzan en garantizar una contrapartida razonable a la lealtad, el compromiso y la productividad que se exige al trabajador: la garantía de su empleabilidad. Es decir, la promesa implícita de que, si pierde su trabajo en la empresa, su nivel de preparación, las habilidades y los conocimientos adquiridos en la empresa le serán útiles cuando necesite buscar un nuevo empleo, de manera que lo mantenga empleable (Fernández-Mateo, 2002).

Cabe señalar también como, en el caso de Europa - y no solo en Europa- los trabajadores asisten al desmantelamiento parcial del Estado de bienestar, con reducciones más o menos maquilladas de las prestaciones por desempleo y las pensiones por jubilación. En la Unión Europea, la Eurocámara aprobó el 20 de noviembre del 2008 un informe en el que se recomendaba a los Estados miembros que se debatiera a escala nacional el retraso de la edad de jubilación y se alentase a los trabajadores a seguir en su empleo, sobre una base voluntaria, hasta alcanzar la edad de jubilación, e incluso más allá de esta. El Gobierno de coalición alemán aprobó en el 2008 retrasar la edad de jubilación a los 67, algo que también ha hecho Dinamarca, además de dar prioridad a aquellos que prolonguen su vida laboral hasta los 70 . También se está retrasando la edad de jubilación en Bélgica, la República Checa y Austria. Otros países como el Reino Unido, Francia o España, también han adoptado este tipo de medidas. Los ejemplos son numerosos, ya que esta es una tendencia generalizada, no solo en los países de la Unión Europea, pues otros países como Israel, Rusia, Australia, Japón o Colombia, han adoptado medidas similares.

Pfeffer (2007) señala como otro gran problema, con efectos muy negativos sobre el compromiso y la productividad, el persistente y creciente conflicto entre trabajo y familia. La progresiva e 
imparable incorporación de la mujer al trabajo, unida a la creciente importancia de nuevos modelos de familia, hace cada vez más difícil la conciliación de la vida laboral y familiar. Si tenemos en cuenta que en un número cada vez mayor de países se encuentran cada vez más mujeres con estudios universitarios con relación a los hombres, que es creciente el número de mujeres que alcanzan puestos de responsabilidad y el número de divorcios y familias monoparentales también va en aumento, la ausencia de medidas que faciliten la conciliación no puede llevar más que al descontento, el estrés y la frustración (Bailyn, 1993; Higgins, Duxbury e Irving, 1992), lo que se traduce en insatisfacción, absentismo y deseo de abandonar la empresa (Boyar, Maertz, Allison, Pearson y Keough 2003; Grandey, Cordeiro y Crouter, 2005; Lourel et al., 2009). En muchos casos, incluso en aquellas empresas en las que se adoptan medidas para la conciliación, se mantienen normas no escritas contra la utilización de esas ventajas, pues poner a la familia antes que al negocio se interpreta como una señal negativa de lealtad y compromiso (Evans, Kunda y Barley, 2004; Perlow, 1997).

De forma simultánea a todos estos hechos, existe una presión constante para que los trabajadores aumenten sus niveles de productividad. Se les exige, en muchos casos, que asuman mayores responsabilidades, una mayor flexibilidad a nivel funcional y una mayor capacidad de aprendizaje y adaptación a cambios cada vez más frecuentes. Cierto es que, al menos en algunos casos, se pueden adoptar medidas que potencien la motivación intrínseca derivada del propio trabajo cuando se buscan estos resultados y esto puede compensar -al menos de forma parcial- esta mayor exigencia, sin afectar de manera negativa la satisfacción laboral. Sin embargo, también es cierto que puede llevar a muchos otros a la percepción de que siempre se les exige más por menos, con efectos devastadores sobre las actitudes hacia la empresa y el trabajo. Por todo esto resulta de crucial importancia articular políticas de motivación capaces de alinear, en la medida de lo posible, los intereses de los trabajadores con los de la propia empresa.

\section{La motivación como función y como proceso}

Existen evidencias de empresas capaces de crear entornos de trabajo especiales en los que los empleados se sienten más satisfechos. Asimismo, existen evidencias de que, al menos algunas, lo consiguen sin sacrificios financieros, e incluso con mejores resultados que los de sus competidores (Cabrera y Bonache, 2002). Como se comenta en la introducción del presente trabajo, son numerosas las investigaciones sobre prácticas de alto rendimiento y alto compromiso que han mostrado relaciones significativas y positivas entre la utilización de este tipo de prácticas y los resultados empresariales. Cabe significar que muchas de estas prácticas, por no decir todas, están relacionadas directa o indirectamente con la motivación.

Por otra parte, según datos del Instituto Great Place to Work, referentes a la lista de las 50 Best Workplaces España 2010, pese a la desfavorable coyuntura económica, las empresas de la lista crecieron un 4,8\% en el 2009, crearon más de 2000 puestos de trabajo y recibieron 250000 solicitudes de empleo, lo que les hizo posible la selección de los mejores talentos para completar sus plantillas. El absentismo laboral en estas empresas fue de tan sólo del 1,94\% frente al 4,7\% de la media nacional (Expansión\&Empleo.com, 2010). Los resultados en otros países en los que se ha llevado a cabo este seguimiento arrojan resultados también positivos. En definitiva, invertir en los empleados, con el propósito de mejorar los niveles de satisfacción y compromiso con la empresa parece ser una inversión rentable.

La función de motivación, entendida como el conjunto de políticas y prácticas de RRHH orientadas a crear las condiciones para atraer, retener y conseguir que los empleados se sientan motivados a contribuir al logro de los objetivos organizativos al utilizar eficaz y eficientemente sus competencias, está llamada a jugar un papel clave en el éxito de la organización. Desde la perspectiva de la dirección, una de las preocupaciones centrales de los directivos debería consistir en averiguar qué es lo que puede impulsar a las personas a actuar conforme a los intereses organizativos de forma sostenida, y qué es lo que puede, y por tanto debe, hacer la organización para sacar todo el provecho posible del potencial y del talento de sus empleados de una manera sostenible.

A fin de comprender cuáles son las posibilidades de intervención de la dirección de RRHH, necesitamos, en primer lugar, comprender el proceso de motivación-satisfacción. Motivación y satisfacción constituyen dos conceptos claves en la relación laboral que se encuentran íntimamente relacionados, pero claramente diferenciados. 


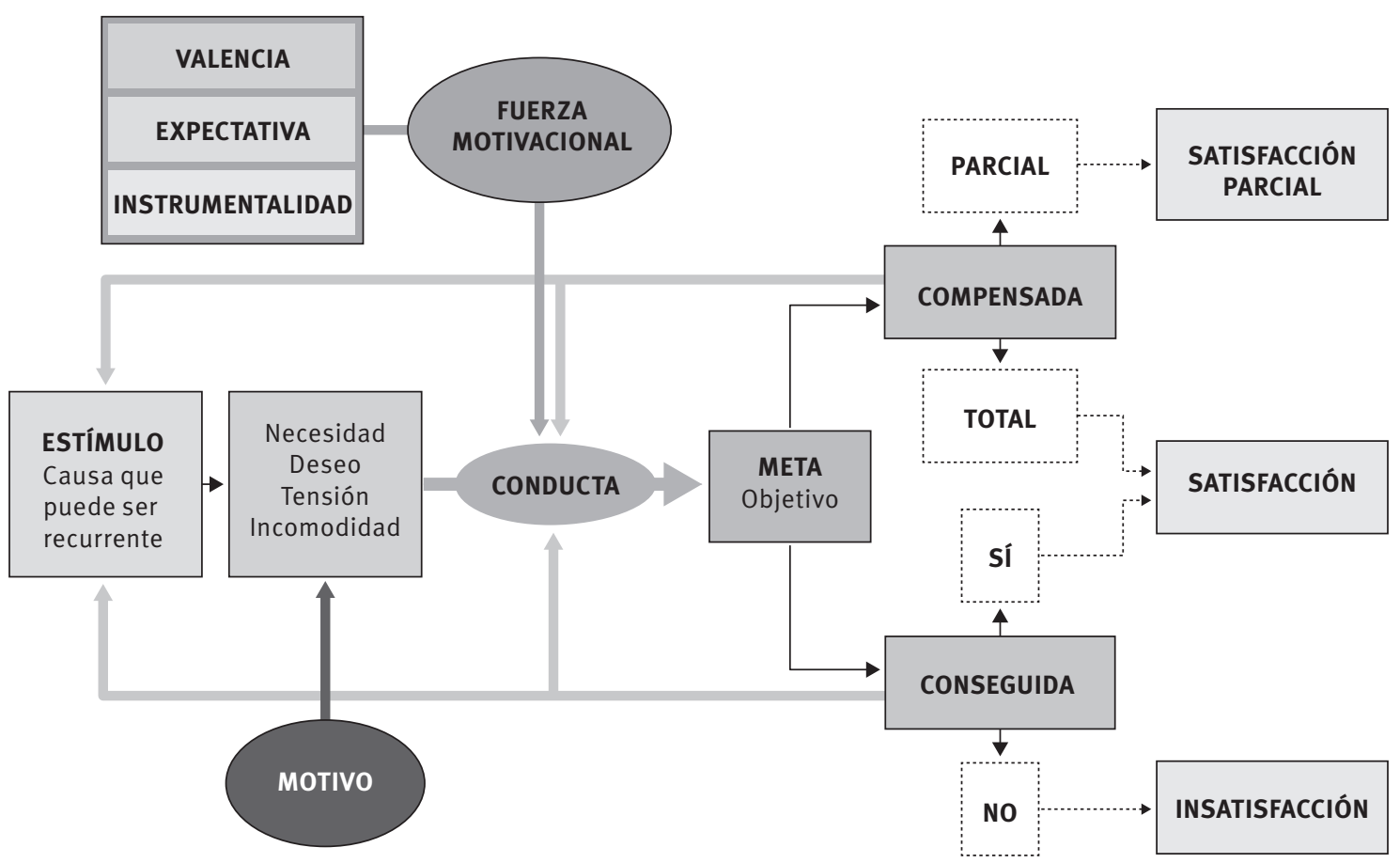

FIGURA 1. Proceso básico de motivación-satisfacción. Fuente: elaboración propia.

Mientras que la motivación en el trabajo hace referencia a disposiciones conductuales que implican la selección, la fuerza y la intensidad de un tipo de comportamiento, la satisfacción constituye un sentimiento frente al trabajo y las consecuencias que de este se derivan para el sujeto (Weinert, 1985). La motivación hace referencia a las aspiraciones de los empleados, mientras que la satisfacción se relaciona con el grado de cumplimento de las aspiraciones. Como se aprecia en la figura 1, la motivación se puede entender como un proceso interno del individuo que desencadena un comportamiento o, cuanto menos, una propensión a actuar orientada a un fin: la satisfacción de una necesidad. La insatisfacción juega el papel de desencadenante del proceso de motivación y la satisfacción es la consecuencia que se espera que tenga el comportamiento motivado (aunque no necesariamente se logrará).

La conducta de los empleados los llevará a obtener o no las recompensas susceptibles de satisfacer las necesidades que fueron origen de la conducta. Sus necesidades se pueden compensar de forma total o parcial cuando reciben una compensación que difiere de la esperada, pero que puede satisfacer al menos parcialmente sus necesidades. Dado que el proceso motivacional es dinámico, existe un proceso de aprendizaje por el cual el nivel de satisfacción alcanzado como resultado de la conducta afecta las conductas futuras y el tipo de estímulos que puede activarlas.

\section{¿Qué hemos aprendido del estudio de la motivación?}

Las teorías de la motivación han analizado el fenómeno desde diferentes perspectivas y con diferentes objetivos. A continuación, se enlistan las que han tenido un mayor impacto en el campo de la dirección de organizaciones y, más concretamente, en la dirección de RRHH.

- Las teorías del contenido representadas por los trabajos de Maslow (1943; 1954), McClelland (1951; 1965), Herzberg (1966; 1968), y Alderfer (1969; 1972), las cuales se centran en el estudio de aquellas variables específicas que motivan a la gente a comportarse de una determinada manera.

- Las teorías del proceso, en sentido estricto, cuyos máximos exponentes son los trabajos de Vroom (1964), y Porter y Lawler (1968); estudian la forma en que las variables que explican la motivación del individuo se relacionan entre sí. 
- Las aportaciones de Hackman y Lawler (1971), y Hakman y Oldham (1976; 1980), las cuales abordan la problemática del diseño y rediseño de puestos y su incidencia sobre la motivación y el comportamiento del individuo.

- La teoría de la equidad (Adams 1963; 1965) que se centra en el efecto sobre la conducta de la percepción de equidad entre esfuerzo y recompensa.

- La teoría de fijación de metas de Locke (1968) y Locke et al. (1981); analiza el impacto que tiene sobre la motivación la fijación de metas concretas y específicas.

- Teorías "no cognoscitivas" u orientadas hacia la modificación del comportamiento organizativo, entre las que se destacan los trabajos de Skinner y sus seguidores: Skinner (1953), Skinner y Rogers (1956), Skinner y Fester (1957), y Skinner y Holland (1970).

- La teoría de la evaluación cognitiva o de la autodeterminación, cuyos mejores exponentes son los trabajos de Deci $(1971 ; 1975)$ y Deci y Ryan (1985); busca determinar la influencia de las recompensas extrínsecas sobre la motivación intrínseca, al partir de la base de que toda recompensa extrínseca tiene un efecto controlador, moldeador del comportamiento, y un efecto informativo en relación con la competencia de este.

En la medida en que excede las pretensiones del presente trabajo, no desarrollaremos las múltiples teorías de la motivación que han surgido a lo largo del tiempo, sino que nos limitaremos a destacar una serie de implicaciones que consideramos relevantes y útiles en la práctica, al tiempo que susceptibles de ser compartidas por la mayoría de los estudiosos del tema:

- Las conductas pueden ser, al menos parcialmente, condicionadas, por lo que aquellas conductas acordes a los intereses de la organización deben reforzarse mientras se tratan de extinguir las conductas contrarias a estos intereses, y reiterar los refuerzos positivos, asociándoles refuerzos negativos o incluso penalizándolas.

- Dado que los individuos difieren en cuanto a las necesidades que son susceptibles de "activarlos", es decir, de impulsarlos o predisponerlos a actuar de un modo determinado, no deberíamos - en la medida de lo posible- reconocerlos, compensarlos y dirigirlos a todos por igual.

- Los factores extrínsecos al trabajo tienen -en muchos casos - un potencial motivador bastante reducido a largo plazo. Más bien se valoran, en la sociedad actual, en términos de condiciones mínimas irrenunciables que actúan como factores insatisfactores o desmotivadores cuando no se encuentran dentro de unos parámetros considerados como aceptables.

- Los factores intrínsecos parecen tener la capacidad de actuar como potentes motivadores, siempre que no interfiera con ellos una mala situación con respecto a los factores extrínsecos citados.

- La fuerza motivacional o predisposición al esfuerzo de un empleado se encuentra determinada por las percepciones de este con relación al valor de las recompensas que puede obtener a cambio de su trabajo (valencia), su propia capacidad para lograr niveles de rendimiento óptimos (expectativa), y la probabilidad de obtener las recompensas esperadas si se alcanza el rendimiento óptimo (instrumentalidad).

- La claridad en la definición de los objetivos y las metas específicas a alcanzar, así como la existencia de una retroalimentación sobre la marcha de los esfuerzos encaminados a conseguir dichos objetivos, están estrechamente relacionadas con el nivel de rendimiento del empleado.

- Sea cual sea el puesto de trabajo y el nivel de responsabilidad de un empleado, es importante que este pueda reconocer la relación que existe entre cualquier forma de reconocimiento otorgado y el rendimiento alcanzado.

- En la medida en que se perciba un desequilibrio o falta de equidad, en términos absolutos o relativos, entre las obligaciones y las responsabilidades a afrontar por un individuo y el nivel de recompensas (tanto intrínsecas como extrínsecas) que se percibe por ello, este tenderá a reestablecer este equilibrio. Si dicho equilibrio no puede ser restablecido por la vía de aumentar las recompensas, lo será por la vía de reducir el esfuerzo y, por tanto, el rendimiento, o el individuo acabará por abandonar la organización en busca de mejores opciones. 


\section{Motivación, satisfacción y posibilidades de intervención}

\section{El marco general}

Si tomamos como marco de referencia la teoría de las expectativas (Isaac, Zerbe y Pitt, 2001; Kopleman y Thompson, 1976; Porter y Lawler, 1968; Vroom, 1964), el tipo de conducta, así como el esfuerzo y la dedicación al trabajo, vienen todos condicionados por la fuerza motivacional. Una fuerza motivacional que es producto de la valencia o el valor que el individuo le asigna a las recompensas que puede obtener si alcanza un nivel de desempeño óptimo, así como de la expectativa o la probabilidad subjetiva que asigna el individuo a que un adecuado nivel de esfuerzo lo lleve a ese desempeño óptimo, y a la instrumentalidad o probabilidad subjetiva que asigna el individuo a que un desempeño óptimo lo lleve al disfrute efectivo de la recompensa.

Como ya hemos señalado, una de las tareas fundamentales de la dirección de RRHH consiste en crear las condiciones adecuadas, por medio de una serie de políticas y prácticas de RRHH necesariamente interrelacionadas, a fin de que los individuos se sientan motivados e implicados en el proyecto de empresa y en el desempeño de las tareas propias de su puesto de trabajo. Esto exige compatibilizar, en la medida de lo posible, la satisfacción de las necesidades individuales con el logro de los objetivos organizativos, como recomienda la teoría de la agencia (Baker, Jensen y Murphy, 1988; Fama, 1991; Jensen, 1994). Las posibilidades de intervención son múltiples, de hecho; si seguimos utilizando el marco de la teoría de las expectativas podemos ver que la dirección puede actuar sobre los tres componentes: valencia, expectativa e instrumentalidad.

Con respecto a la valencia, la dirección de RRHH debe preocuparse por evaluar, de forma continuada, la idoneidad del sistema de recompensas (tanto intrínsecas como extrínsecas) ofrecido por la organización, al utilizar como información clave las necesidades y las preferencias de los empleados. Resulta obvio que las personas difieren en lo que respecta a la motivación, pero, además, lo que ahora motiva a un individuo, no lo hará necesariamente en el futuro. Por esto debemos tratar de conocer cuáles son las metas que desean alcanzar los individuos, cuáles son sus prioridades y cuál es su nivel de satisfacción respecto a las distintas dimensiones relevantes del puesto de trabajo. Esta información resulta fundamental para introducir cambios efectivos en el sistema de recompensas.

En relación a la expectativa, cabe señalar que, debido a un proceso de aprendizaje, es previsible que esta aumente con el paso del tiempo, en la medida en que el empleado perciba que un mayor nivel de esfuerzo o dedicación a la tarea se traduce en un mejor desempeño o resultado de primer nivel. Dado que la relación entre esfuerzo y desempeño va a depender, fundamentalmente, de una adecuada percepción del puesto y de las habilidades y capacidades del individuo, se plantean distintas posibilidades de intervención.

En primer lugar, la percepción del puesto puede resultar más ajustada a las necesidades organizativas si el empleado cuenta con una descripción del puesto y de sus responsabilidades precisa y actualizada. También jugará un papel importante la fijación de objetivos concretos y específicos, susceptibles de orientar al empleado hacia las conductas efectivas, tal como recomienda la teoría de la fijación de metas (Latham y Locke, 2002; Latahm y Yukl, 1975; Locke, 1968; Locke et al. 1981). En este sentido, resulta imprescindible llevar a cabo procesos de evaluación del desempeño capaces de proporcionar una adecuada y oportuna retroalimentación al empleado (Tziner, Kopelman y Neomi, 1993). Una actitud abierta al diálogo, unida a buenas dotes de comunicación y de coaching por parte de los mandos intermedios, resultará asimismo de gran valor.

En segundo lugar, las habilidades y las capacidades necesarias para garantizar el resultado de primer nivel deben garantizarse al inicio de la relación laboral, por medio de las funciones de reclutamiento, selección e integración; asimismo, durante la permanencia del empleado en la organización, por medio de la actualización de sus competencias - en función de las necesidades de la organización-, de la que han de ocuparse la formación y el desarrollo. Todo esto garantizará una adecuación continua entre el perfil de competencias exigidas por el puesto y las del empleado.

Por último, con respecto a la instrumentalidad, la cual relaciona el desempeño o resultado de primer nivel con las recompensas o resultados de segundo nivel, cabe diferenciar entre las recompensas intrínsecas y las extrínsecas. Las recompensas intrínsecas, es decir, las que tienen que ver con la propia realización de un trabajo más 
o menos enriquecido (Hackman y Lawler, 1971; Hakman y Oldham, 1976; 1980), pueden considerarse recompensas autoconcedidas. Por tanto, el papel de la organización ha de centrarse, fundamentalmente, en alcanzar el mejor ajuste posible entre las necesidades del individuo, los condicionantes que plantean los procesos de producción y la estructura en general de la organización, así como las características de los puestos de trabajo. Lo anterior en procura, eso sí, de hacer patente de forma explícita y clara el valor intrínseco del trabajo desarrollado.

En lo que respecta a las recompensas extrínsecas (las que se reciben como contrapartida al trabajo), resulta fundamental conseguir una elevada percepción de equidad tanto interna como externa, por parte de los empleados (Adams, 1963; 1965). En este propósito resultan elementos indispensables la transparencia en la actuación de la organización, la adecuada valoración de los puestos y la existencia de sistemas de evaluación del desempeño objetivos y eficaces. Estos sistemas se definen con la participación de los afectados, quienes han de ser conocedores de los criterios y los métodos a utilizar, así como de las consecuencias que se van a derivar de la evaluación. La entrevista de evaluación y los procedimientos de apelación pueden jugar también un papel fundamental a la hora de ganar credibilidad, corregir conductas y resolver dudas y temores de los empleados.
En definitiva, alcanzar elevados niveles de motivación y satisfacción en los empleados implica, en la práctica, a todas las funciones del área de RRHH, una cuestión sobre la que se insiste en el último apartado de este trabajo. A continuación, nos centramos en la identificación de las necesidades y las prioridades de los empleados para un adecuado diseño del sistema de recompensas de la organización, sin perder de vista las prioridades de esta.

\section{Necesidades, preferencias y satisfacción}

El comportamiento humano depende de un número muy importante de factores, algunos de ellos controlables, pero otros no. Entre los segundos, se encuentran el sistema de recompensas de una empresa, el cual Baron y Kreps (1999) señalan como el mayor impulsor de la motivación humana. Desde la concepción de las escuelas clásicas que planteaban la motivación como una cuestión exclusivamente económica, hasta la actualidad, el abanico de factores considerados como motivadores potenciales en la relación laboral se ha ampliado de forma paulatina. Maier (1963) destaca cómo - desde la década de los treinta- las investigaciones confirmaban la importancia de los factores no económicos como motivadores.

En la actualidad, en la ecuación de la motivación podemos incluir factores como, por ejemplo,

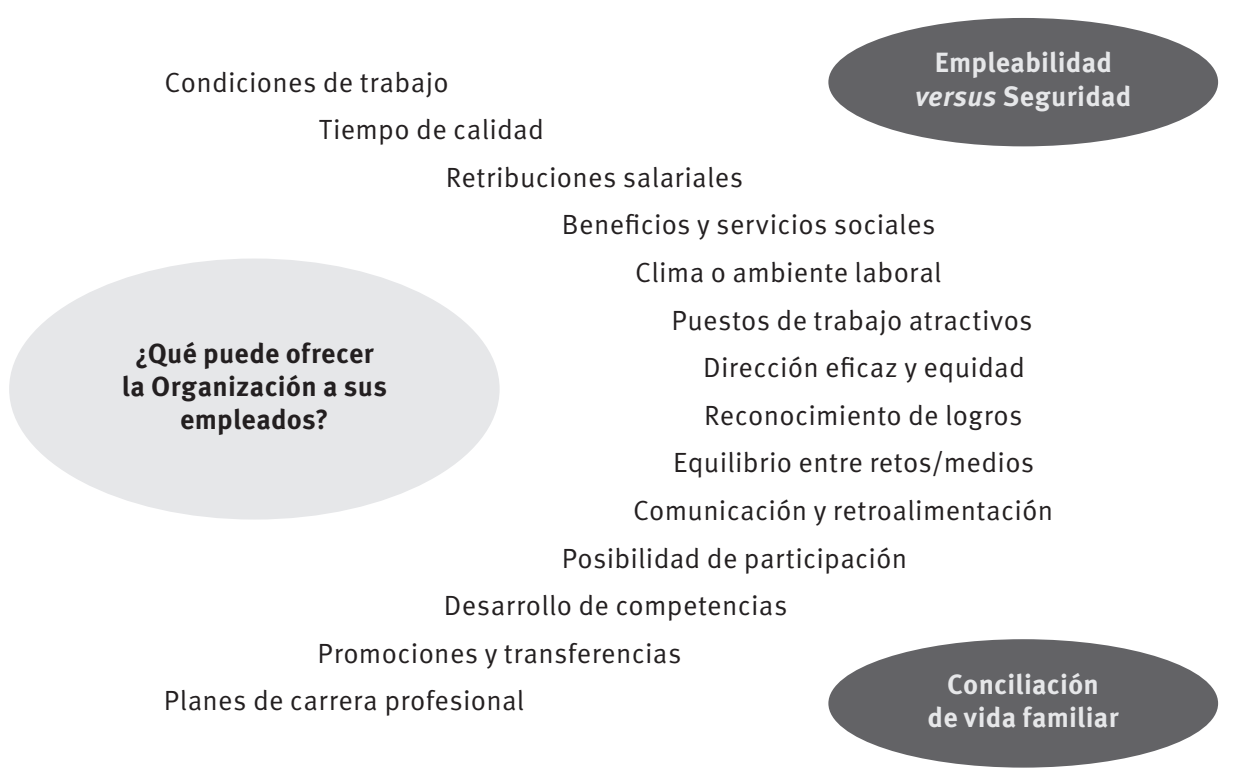


el estilo de liderazgo, las posibilidades de participación, la garantía de empleabilidad, la carrera profesional y las posibilidades de formación y desarrollo, la propia reputación de la empresa, etc. Por tanto, al hablar de sistema de recompensas, adoptamos una concepción muy amplia que incluye todos aquellos factores susceptibles de tener un impacto significativo en la motivación de los empleados al cubrir cualquiera de sus necesidades por medio de la relación de trabajo.

Especial atención merecen las recompensas intrínsecas, directamente vinculadas con el contenido del trabajo. Autores como Deci y Ryan (1987 y 1992) consideran que la motivación intrínseca es una de las formas más poderosas de motivación porque se encuentra asociada con el incremento del desempeño, la predisposición a implicarse en otras tareas, la mejora del pensamiento creativo y la mejora en la salud física y psicológica, así como en la memoria. Una tarea intrínsecamente motivadora debe incluir claridad en los objetivos, debe ser razonablemente retadora y proporcionar retroalimentación inmediata sobre los progresos (Amabile, Hill, Hennesey y Tighe, 1994; Elliot y Harckiewicz, 1994). También es necesario, a fin de conseguir esta motivación intrínseca, que el individuo se considere competente o, al menos, capaz de aprender lo necesario, a fin de que esté en capacidad de alcanzar los objetivos del puesto y obtenga una percepción de control y de libre elección en relación con lo que hace (Deci y Ryan, 1987; 1992).

Como se señaló, la dirección de RRHH tiene como una de sus funciones esenciales el diseño de un sistema de evaluación y recompensa capaz de alinear los intereses individuales con los organizativos y evitar comportamientos oportunistas, pues este es el principal instrumento con el que cuenta para tratar de influir en las actitudes y orientar la conducta de los empleados hacia comportamientos productivos. Además, ha de hacerlo teniendo en cuenta el valor y la singularidad (escasez y especificidad) del capital humano de la organización (Lepak y Snell, 1999 y 2002), es decir, el potencial de este capital humano para mejorar la eficacia y eficiencia de la empresa (Barney, 1991; Ulrich y Lake, 1991). Obviamente, la empresa debería poner mayor énfasis en satisfacer las necesidades de aquellos miembros de la organización que cuentan con competencias esenciales (core competencies en términos de Hamel y Prahalad, 1995), quienes pueden constituir la fuente de ventajas competitivas y que, además, son difíciles de encontrar en el mercado laboral y costosos de capacitar; son empleados con los que se deben establecer relaciones a largo plazo y basadas en el compromiso (Lepak y Snell, 1999).

Conseguir lo anterior hace imprescindible la detección y actualización periódica de las necesidades y prioridades de los empleados, puesto que las personas no solo difieren con respecto a estas necesidades y prioridades, sino que cambian o, cuanto menos, evolucionan a lo largo del tiempo cuando varían sus circunstancias personales o familiares, sus expectativas, valores, hábitos, etc. Solo de este modo es posible evaluar la idoneidad del sistema de recompensas utilizado por la organización y hacer los ajustes necesarios. En la actualidad, el medio más utilizado para diagnosticar la situación a que se enfrenta una organización respecto a la motivación-satisfacción de sus empleados son las encuestas de satisfacción laboral. En muchos casos, estas encuestas se basan en instrumentos ya validados, entre los cuales se destacan el Job Descriptive Index (Smith, Kendall y Hullin, 1969) y el Minnesota Satisfaction Questionaire (Weiss, Dawis, England y Lofquist, 1977). En otros casos se utilizan cuestionarios específicamente desarrollados de acuerdo con las peculiaridades de la empresa, pero cuya validez y fiabilidad estadísticas no suelen ser contrastadas de forma debida. Además, estos cuestionarios de empresa plantean la desventaja adicional de no permitir comparaciones con una norma, imprescindibles para la adecuada interpretación de los resultados (Saari y Judge, 2004).

Una vez se identifican esas necesidades, las empresas deben decidir qué colectivos han de recibir una atención preferente. En la medida de lo posible, las empresas deben realizar esfuerzos encaminados a individualizar o personalizar, al menos parcialmente, sus sistemas de recompensas. La constatación de que existen trabajadores más sensibles que otros a reconocimientos de tipo verbal, quienes tienen mayores necesidades informativas o de participación, valoran más la posibilidad de trabajar con una cierta autonomía o tienen preferencias por determinado tipo de prestaciones en especie, ofrece posibilidades para esta individualización de las recompensas. No obstante, llevar al extremo esta posibilidad puede conducir a una excesiva complejidad del sistema de incentivos, a costes de administración del sistema insostenibles e, incluso, a la percepción 
de inequidad si no se gestiona de forma adecuada. Por tanto, es conveniente tratar de identificar perfiles motivacionales más o menos homogéneos que permitan ofrecer sistemas flexibles, pero no demasiado complejos.

Por otra parte, existe otra vía de actuación relacionada con los procedimientos de reclutamiento y selección. Los responsables de la función de RRHH deben realizar esfuerzos a fin de tratar de evaluar las necesidades y expectativas de los candidatos a formar parte de la organización, y así evitar integrar candidatos cuyas necesidades y expectativas no van a poder ser nunca cubiertas por medio de las recompensas y condiciones de trabajo que la empresa puede ofrecer. Evidentemente, las organizaciones también tienen que respetar la máxima de no crear falsas expectativas en los empleados, con tal de resolver sus necesidades de personal a corto plazo, pues esto es una garantía de frustración e insatisfacción laboral futura que puede acarrear consecuencias negativas en forma de bajo rendimiento, mala calidad del servicio, conflicto, absentismo, rotación, etc.

\section{La necesidad de una perspectiva estratégica de la motivación}

En este apartado se explica qué conlleva adoptar lo que hemos denominado "una perspectiva estratégica de la motivación". En primer lugar, exige tener en cuenta la situación particular de una empresa, sus objetivos y su estrategia organizativa en las políticas y prácticas de motivación. Esto implica que la misión, la visión y los objetivos estratégicos de la empresa han de guiar la toma de decisiones en materia de políticas y prácticas de motivación y, más específicamente, en la selección de recompensas. Como ha mostrado ampliamente la literatura, las prácticas de RRHH en general, y las de motivación en particular, deben variar en función de la estrategia de la compañía (Albizu y Landeta, 2001; Miles y Snow, 1984; Schuler, 1987; Schuler y Jackson, 1987). Los requerimientos que plantea y las oportunidades que ofrece -en lo relativo a los RRHH - una estrategia de crecimiento dinámico frente a una estrategia de cosecha o desinversión, o las implicaciones de basar los

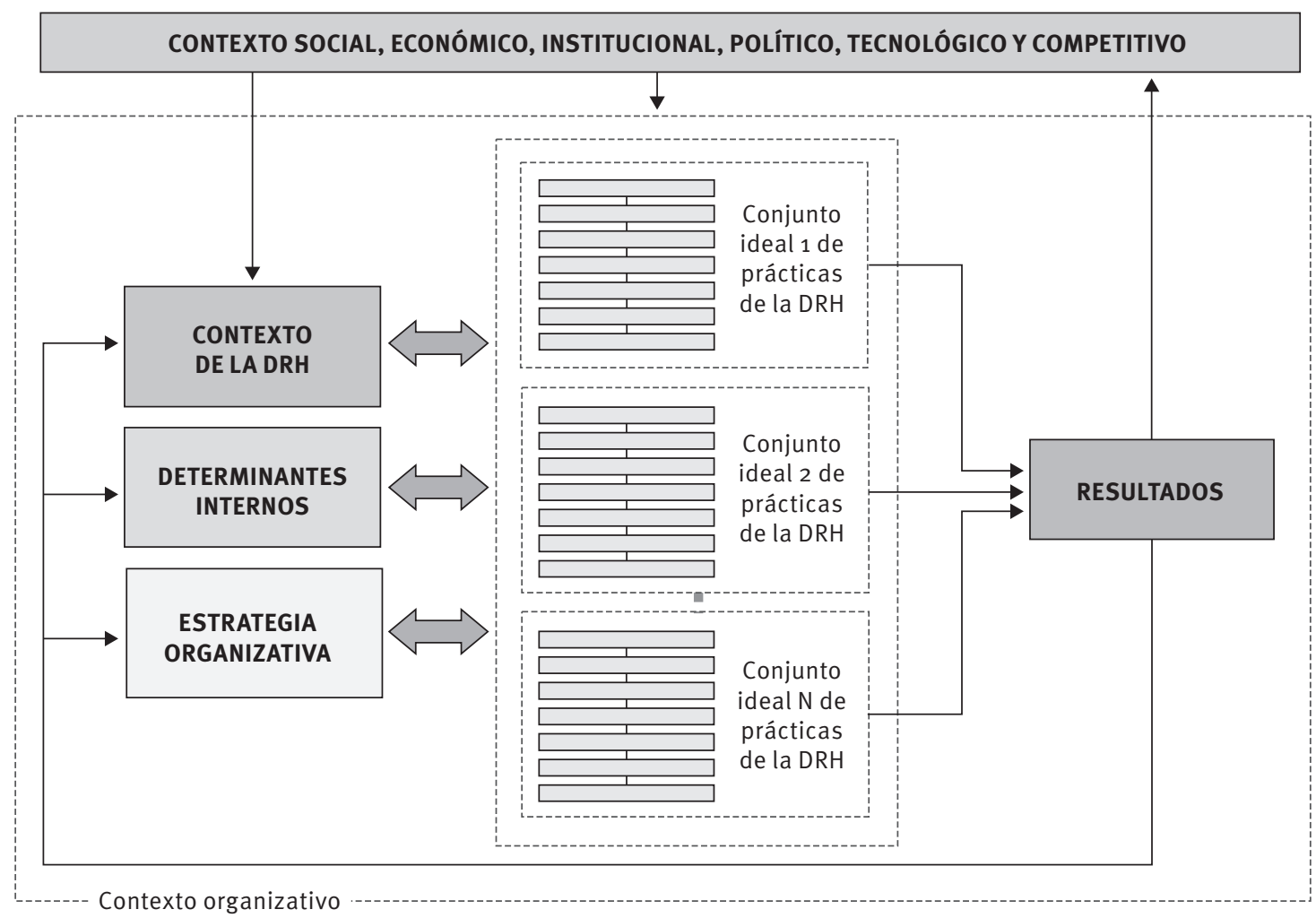

Contexto externo

FIGURA 3. La dirección de recursos humanos desde la perspectiva contextual. Fuente: Adaptado de MartínAlcázar, F., Romero-Fernández, P. M. y Sánchez-Gardey, G. (2005). 
argumentos competitivos en la innovación, la calidad o la reducción de costes, son completamente diferentes.

Ahora bien, lo difícil es actuar en consecuencia en un tema tan sensible, el cual afecta de forma tan directa el bienestar de los individuos, y además se encuentra tan sujeto a presiones institucionales derivadas de la costumbre, de las prácticas aceptadas y "sindicalizadas", de las limitaciones que marcan las normas legales, de las modas que derivan de las grandes recetas de reputados expertos o de las prácticas de los líderes de la industria. Todo esto aderezado en una atmósfera en la que, por una parte, la mayoría se adhiere al discurso de la importancia clave de los RRHH para garantizar la competitividad de la empresa y, por otra, de facto, sigue actuando desde una óptica de coste de personal y no de inversión en capital humano.

No obstante, a fin de que las prácticas de motivación de la empresa, y —más concretamente- sus sistemas de recompensas, cumplan su cometido, los responsables no deberían limitarse a la operatoria cotidiana y dejar de tomar las decisiones apropiadas a nivel estratégico, solo por no romper con la dinámica de un sistema ya establecido. Tampoco deberían adherirse sin más a la "norma" del sector o al ámbito de actuación, ni ceder en demasía otro tipo de presiones institucionales en busca de legitimidad. Deben adoptarse políticas de motivación flexibles y contingentes, pues lo mejor no existe, si no lo más adecuado para la situación y los objetivos concretos de la empresa en cada momento de su vida, lo que exige capacidad para reinventar los mecanismos utilizados para atraer, retener y motivar los RRHH, en función de la evolución del contexto y sus propias necesidades.

En segundo lugar, una perspectiva estratégica conlleva adoptar políticas que tengan en cuenta la composición de la plantilla actual, sus necesidades, las preferencias y también sus diferencias. Al respecto, Lepak y Snell (1999, p. 42) señalan, por ejemplo, que "puede resultar demasiado simplista asumir que un tipo de relación de empleo o un único conjunto de prácticas de RRHH funcionará para todos los empleados". Plantean que las empresas obtendrán mejores resultados utilizando prácticas de RRHH diferenciadas para distintos colectivos, en función del valor que aportan a la empresa y la singularidad de sus competencias o habilidades, pues es razonable establecer diferentes relaciones de empleo con esos diferentes colectivos. Herrera (2001, p. 76) introduce el concepto de unidades de RRHH que define como "aquél colectivo o grupo de colectivos para los que la empresa decide plantear una estrategia de dirección de recursos humanos diferenciada de la que aplicará al resto de la organización". Mediante este concepto pretende significar que es razonable e incluso conveniente que las empresas planteen e implanten diferentes políticas y prácticas de RRHH para diferentes colectivos.

Por su parte, Cabrera y Gómez-Mejía (2002, p. 20) señalan que no solo "la dirección estratégica de RRHH debe guiarse por la coherencia interna entre unas prácticas y otras, y la coherencia con el sistema de prácticas y las competencias esenciales que marca la estrategia de la organización", sino que "las empresas han de ser capaces de manejar relaciones de empleo múltiples con diferentes grupos de empleados. Algunas de estas relaciones serán de larga duración y se basarán en el compromiso. Otras serán relaciones de mercado basadas en el cumplimiento de un contrato, y algunas más consistirán en asociaciones de beneficio mutuo". Esto supone la existencia de diferentes políticas de RRHH para diferentes colectivos de empleados en función del valor estratégico que aportan a la organización, y la mayor o menor facilidad para encontrar las competencias necesarias en el mercado laboral, en concordancia con lo propuesto por Lepak y Snell (1999).

Puede resultar chocante que a lo largo de nuestro discurso comenzamos hablando de políticas y prácticas de motivación para pasar a hablar de relaciones de empleo asociadas a sistemas de prácticas de RRHH, más que específicamente a prácticas de motivación. El motivo de hacerlo radica en el hecho de que no pueden desvincularse las prácticas de motivación de las demás prácticas de RRHH, pues es necesario alcanzar un alto grado de consistencia interna entre las prácticas, además de la coherencia externa con la estrategia y los otros factores contextuales relevantes. Asimismo, como señalamos al analizar el proceso de motivación desde la perspectiva de la teoría de las expectativas, las demás subfunciones de RRHH (reclutamiento, selección e integración, formación y desarrollo, evaluación del desempeño, valoración de puestos y competencias, gestión del sistema de información de RRHH, comunicación interna, etc.) juegan en ese proceso un papel crucial para que deriven resultados satisfactorios tanto para la empresa como para sus empleados. Por tanto, el tercer elemento de la perspectiva estratégica de la motivación 
lo constituye esa visión integral de las funciones de RRHH, cada una de las cuales debe jugar su papel a la hora de conseguir la implicación de los empleados. Así, a modo de ejemplo,

- La supervisión y los procesos de integración e información-comunicación, deben garantizar que se fijen objetivos claros, medibles, alcanzables pero retadores, así como facilitar la participación y permitir al empleado identificar con claridad el rol a desempeñar y que faciliten establecer expectativas claras.

- El diseño de los puestos de trabajo debe resultar satisfactorio, no solo desde el punto de vista técnico, sino también del social, en atención al potencial de motivación intrínseco del contenido del trabajo.

- El análisis y la valoración de puestos y competencias debe constituir un elemento que permita definir un ajuste persona-puesto adecuado, así como un instrumento para garantizar la equidad interna.

- Los procesos de reclutamiento, selección e integración o socialización deben garantizar que se integren en la organización personas con competencias valiosas para la empresa, a las cuales se puedan ofrecer recompensas en su sentido más amplio- que puedan satisfacer razonablemente sus necesidades.

- Los inventarios de habilidades deben permitirnos reconocer el valor del capital humano de la organización, de modo que permitan recompensas a cada cual según su valía. También deben facilitar la adopción de medidas específicas para retener y ampliar esas habilidades, o bien adquirir otras y permitir aclarar expectativas de futuro, así como orientar carreras profesionales.

- La formación y el desarrollo debe satisfacer las necesidades de la empresa, pero también pueden alentar la implicación y el compromiso, reducir la ansiedad, aumentar la confianza y acercar a los empleados a nuevas recompensas y a garantizar su empleabilidad.

- La investigación del mercado laboral debe constituir un instrumento para garantizar la equidad externa. Las empresas no pueden olvidar que también compiten en el mercado de recursos y, si bien pueden aprovecharse de ciertas imperfecciones del mercado laboral, las habilidades y el talento tienen un precio.
- La evaluación del desempeño debe servir como instrumento para la justa recompensa a aquellos que logran los objetivos y adoptan las conductas apropiadas, así como para facilitar el desarrollo profesional de los empleados y mejorar, en lugar de empeorar, la comunicación y las relaciones entre directivos y empleados.

\section{Conclusiones}

El presente trabajo presenta la motivación como un proceso cuyo output fundamental es la sensación de satisfacción o insatisfacción con el trabajo, la actitud o el sentimiento que tiene, como ha demostrado insistentemente la literatura, consecuencias relevantes en otras variables: el compromiso organizativo, el comportamiento cooperativo, la calidad del trabajo realizado, y los niveles de absentismo y de rotación laboral. En definitiva, dado el conjunto de "motivos" o recompensas que ofrece la empresa a los empleados a cambio de su trabajo, de la forma de gestionarlas va a depender, en gran medida, el comportamiento de ellos.

Al tomar como modelo de referencia la teoría de las expectativas, resulta, como hemos podido constatar, que las políticas y prácticas de RRHH pueden impactar tanto en la valencia como en la expectativa y la instrumentalidad, pues las elecciones y las actuaciones en materia de RRHH pueden afectar tanto el valor subjetivo asignado al sistema de compensación ofrecido como a la percepción subjetiva de la capacidad de cada individuo de alcanzar las metas establecidas, en razón a la equidad percibida en el logro de las recompensas prometidas.

Además, este modelo ofrece una perspectiva más amplia e integrada de las posibilidades de intervención de la dirección de la empresa con el fin de influir en la conducta de los empleados desde una perspectiva dinámica orientada tanto a corto como largo plazo. Queda muy claro que la motivación y la satisfacción laboral de los empleados no es un tema vinculado, ni exclusiva, ni - me atrevería a decir-, fundamentalmente, a la configuración de un atractivo sistema salarial o de compensación, en general, sino que implica directa o indirectamente a todas las funciones de área de RRHH. El propio modelo nos pone sobre la pista de un buen número de posibles interacciones 
o interdependencias entre diferentes políticas y prácticas de RRHH.

El reconocimiento de estas interacciones o interdependencias obliga, por tanto, a adoptar una perspectiva sistémica que lleve a la configuración de un sistema de prácticas de RRHH internamente consistentes entre sí, orientadas a atraer, retener y motivar a los empleados. No obstante, teniendo en cuenta que no todos los empleados aportan un capital humano igualmente valioso para la empresa y difícil de adquirir, es razonable que estas prácticas varíen de un colectivo a otro o, cuanto menos, se apliquen con diferente nivel de intensidad. En todo caso, si se aplica este sistema "elitista", debe evitarse que las consecuencias de una intensa inequidad percibida (entre los distintos colectivos) conlleve unas consecuencias sobre su conducta que eliminen las ventajas de costes que puedan derivar de no aplicar a todos los empleados un sistema de prácticas que la literatura suele denominar, en sus acepciones más comunes, "de alto desempeño" o "de alto compromiso".

Obviamente, desde una perspectiva sistémica no se puede olvidar que la empresa es un sistema abierto y, por tanto, a la hora de definir esos sistemas de prácticas de RRHH de la empresa, en general, así como el conjunto de prácticas de compensación en particular, va a ser absolutamente necesario tener en cuenta el contexto en el que desarrolla su actividad la empresa en cuestión. Las empresas compiten tanto en los mercados de productos-servicios como en los mercados de factores, entre ellos, el mercado laboral. Por tanto, han de tener en cuenta a la hora de tomar sus decisiones en el ámbito de los RRHH factores como, por ejemplo, la evolución de la economía, la tasa de desempleo, la escasez o la abundancia de recursos específicos, el poder sindical, la cultura, las prácticas de otras empresas y, en especial, los líderes del sector, etc.

Desde una perspectiva estratégica, la empresa debe tener siempre como punto de referencia qué tipo de empresa quiere ser, dónde y con quién quiere competir, así como qué tipo de ventajas competitivas quiere hacer valer. Por tanto, sus prácticas de atracción, retención y motivación de talento humano deben estar orientadas a facilitar que se haga realidad esta visión estratégica, la cual debería ser, en la medida de lo posible, una visión conocida y compartida por los miembros de la organización. Desde la dirección de RRHH se debe trabajar para alinear los intereses de los empleados y la organización, así como con el fin de atraer, retener y motivar el talento humano necesario para sustentar, por medio de sus competencias individuales, las core competences sobre las que va a basar sus ventajas competitivas. Por tanto, su papel es clave en la competitividad de la organización, especialmente en sectores intensivos en conocimiento que dependen todavía más del talento de sus empleados que el resto de sectores productivos.

Finalmente, señalar que si bien el presente trabajo ofrece algunos lineamientos generales sobre cómo pueden afrontar los directivos el reto de atraer, retener y motivar a sus empleados, la concreción de las medidas a adoptar exige un trabajo de análisis y reflexión, para lo cual es necesario que cuenten con las herramientas y los procesos adecuados. Por esto, consideramos que es necesario seguir investigando en esta línea desde una perspectiva práctica y aplicada, orientada al desarrollo de protocolos e instrumentos que faciliten el diagnóstico de las necesidades organizativas e individuales. Asimismo, es necesario profundizar en el análisis de las interrelaciones entre las diferentes prácticas de $\mathrm{RRHH}$, con el objetivo de facilitar el diseño de sistemas integrados de prácticas que se ajusten a las circunstancias y a las necesidades particulares de cada organización.

\section{Referencias}

Adams, J. S. (1963). Toward an understanding of inequity. Journal of Abnormal and Social Psychology, 67(5), 422-436.

Adams, J. S. (1965). Inequity in social exchange. In Advances in experimental social psychology (Vol. 2, pp. 267-299). New York: Academic Press.

Akerlof, G., Rose, A. y Yellen, J. (1988). Job switching and job satisfaction in the us labor market. Brookings Papers on Economic Activity, 2, 495-582.

Albizu, E. y Landeta, J. (2001). Dirección estratégica de recursos humanos. Teoría y práctica. Madrid: Pirámide,

Alderfer, C. P. (1969). An empirical test of a new theory of human needs. Organizational Behavior and Human Performance, 4(2), 142-175.

Alderfer, C. P. (1972). Existence, relatedness, \& growth. Nueva York: Free Press.

Amabile, T. M., Hill, K. G., Hennesey, B. A. y Tighe, E. M. (1994). The Work Preference Inventory: Assessing intrinsic and extrinsic motivation orientations. Journal of Personality and Social Psychology, 66(5), 950-967.

Bacharach, S. B., Bamberger, P. y Conley, S. (1991). Work-home conflict among nurses and engineers: 
Mediating the impact role stress on burnout and satisfaction at work. Journal of Organizational Behavior, 12(1), 39-53.

Bailyn, L. (1993). Breaking the molde; women, men, and time in the new corporate world. Nueva York: Free Press.

Baker, G., Jensen, M. y Murphy, K. (1988). Compensation and incentives: Practice vs. theory. The Journal of Finance, 43(3), 593-616.

Baldwin, R. E. y Robert-Nicoud, F. (2000). Free trade agreements without delocation. Canadian Journal of Economics, 33(3), 766-786.

Barney, J. (1991). Firm resources and sustained competitive advantage. Journal of Management, 17(1), 99-120.

Barney, J. B. y Wright, P. M. (1998). On becoming a strategic partner: The role of human resources in competitive advantage. Human Resource Management, 37(1), 31-46.

Baron, J. N. y Kreps, D. M. (1999). Strategic Human Resources. Frameworks for general managers. Nueva York: John Wiley \& Sons.

Bateman, T. S. y Organ, D. W. (1983). Job satisfaction and the good soldier: The relationship between affect and citizenship. Academy of Management Journal, 26(4), 587-595.

Becker B. E. y Huselid M. A. (1998). High performance work systems and firm performance: A synthesis of research and managerial implications. Research in Personnel and Human Resources Management, 16, 53-101.

Becker, B. E. y Gerhart, B. (1996). The impact of resource management on organizational performance: progress and prospects. Academy of Management Journal, 39(4), 779-801.

Begley, T. y Czajka, J. (1993). Panel analysis of the moderating effects of commitment on job satisfaction, intent to quit, and health following organizational change. Journal of Applied Psychology, 78(4), 552556.

Blau, G. (1993). Further exploring relationship between job search and voluntary individual turnover. Personnel Psychology, 46(2), 313-330.

Bluedorn, A. C. (1982). A unified model of turnover from organizations. Human Relations, 35(2), 135153.

Boxall, P. (1996). The strategic human resource management debate and the resource-based view of the firm. Human Resource Management Journal, 6(3), 59-75.

Boyar, S. L., Maertz, C. P., Allison, J., Pearson, W. y Keough, S. (2003). Work-family conflict: A model of linkages between work and family domain variables and turnover intentions. Journal of Managerial Issues, 15(2), 175-190.

Brown, A. y Mitchell, T. (1993). Organizational Obstacles: links with financial performance, customer satisfaction, and job satisfaction in a service environment. Human Relations, 46(6), 725-757.

Bureau of Economic Analysis. (2011). International economic accounts. Bea.gov. Recuperado de http://bea.gov/international/di1usdop.htm

Cabrera, A. y Bonache, J. (2002). Recursos humanos y ventaja competitiva. En J. Bonache y A. Cabrera (Eds.), Dirección estratégica de personas. Evidencias y perspectiva para el siglo XXI (pp. 3-26). Madrid: Prentice Hall.

Cabrera, A. y Gómez-Mejía, L. (2002, julio/septiembre). La sexta fuerza competitiva: ¿cómo gestionar a las personas en al siglo XXI?. Revista de Empresa, 1, 17-27.

Cameron, K. (1994). Strategies for Successful Organizational Downsizing. Human Resource Management, 33(2), 189-211.

Capelli, P. (2006). Changing career paths and their implications. En E. E. Lawler III y J. O’Toole (Eds.), America at work: Choices and challenges. Nueva York: Palgrave Macmillan, 211-224.

Cascio, F. R. (1991). Managing human resource: Productivity, quality of work life and profits ( $3^{\mathrm{a}}$ edición). Nueva York: McGraw-Hill.

Cascio, W. F. 1991. Costing human resource: The financial impact of behavior in organizations. Boston: PWS.

Cazes, S. y Nesporova, A. (2007). Flexicurity. A relevant approach in Central and Eastern Europe. Geneva: International Labour Office.

Alcelay, S. y Perait, L. (2010, febrero 28). Un millón de empleados faltan cada día al trabajo en España. ABC.es. Recuperado de http://www.abc. es/20100228/economia-laboral/millon-empleados-faltan-cada-20100228.html

Clegg, C. W. (1983). Psychology of employee lateness, absence, and turnover: a methodological critique and an empirical study. Journal of Applied Psychology, 68(1), 88-101.

Conference Board. (2005, febrero 28). U. S. job satisfaction keeps falling. The Conference Board Reports today. News release.

Dalton D. y Mesch, D. (1991). On the extent and reduction of avoidable absenteeism: An assessment of absence policy provisions. Journal of Applied Psychology, 76(6), 810-817.

Deci, E. L. (1971). Effects of externally mediated rewards on intrinsic motivation. Journal of Personality and Social Psychology, 18(1), 105-115.

Deci, E. L. (1975). Intrinsic motivation. Nueva York: Plenum Publishing Corp.

Deci, E. L. y Ryan, R. M. (1985). The general causality orientations scale: Selfdetermination in personality. Journal of Research in Personality, 19, 109-134.

Deci, E. L. y Ryan, R. M. (1987). The support of autonomy and the control of behavior. Journal of Personality and Social Psychology, 53(6), 1024-1037. 
Deci, E. L. y Ryan, R. M. (1992). The initiation and regulation on intrinsically motivated learning and achievement. En A. K. Boggiana y T. S. Pittman, (Eds.), Achievement and motivation (pp. 9-36). Nueva York: Cambridge University Press.

Delaney, J. T. y Huselid, M. A. (1996). The impact of human resource management practices on perceptions of organizational performance. Academy of Management Journal, 39(4), 949-969.

Deloitte Research. (2004). It's 2008: Do you know where your talent is? Nueva York: Deloitte Research.

Elliot, A. J. y Harckiewicz, J. M. (1994). Goal setting, achievement orientation and intrinsic motivation: A mediational analysis. Journal of Personality and Social Psychology, 66(5), 968-980.

Evans, J. A., Kunda, G. y Barley, S. R. (2004). Beach time, bridge time, and billable hours: The temporal structure of technical contracting. Administrative Science Quarterly, 49(1), 1-38.

Expansión\&Empleo.com. (2010). Vodafone, Louis Vuitton y Cisco entre las mejores empresas para trabajar. Expansionyempleo.com. Recuperado de http:// archivo.expansionyempleo.com/2010/04/22/ desarrollo_de_carrera/1271927006.html

Fama, E. (1991). Time, salary, and incentives payoffs in labor contracts. Journal of Labor Economics, 9, 25-44.

Farh, J., Podsakoff, P. M. y Organ, D. W. (1990). Accounting for organizational citizenship behavior: Leader fairness and task scope versus satisfaction. Journal of Management, 16(4), 705-721.

Fernández-Mateo, I. (2002). La gestión de carreras. En J. Bonache y A. Cabrera (Eds.), Dirección estratégica de personas. Evidencias y perspectiva para el siglo XXI. (pp. 231-262). Madrid: Prentice Hall.

Flamholtz, E. G. y Lacey, J. (1981). The implications of the economic theory of human capital for personnel management. Personnel Review, 10(1), 30-39.

Fosam, E. B., Grimsley, M. F. J. y Wisher, S. J. (1998). Exploring models for employee satisfaction: with particular reference to a police force. Total Quality Management, 9(2-3), 235-247.

Francis, J. D. y Pett, T. L. (2004). Retrenchment in declining organizations: Towards an integrative understanding. Journal of Business and Management, 10(1), 39-52.

Frauenheim, E. (2006, febrero). Studies: More workers look to switch jobs. Workforce Management, 13, 12.

Fugate, M., Kinicki, A. J., y Ashforth, B. E. (2004). Employability: A psycho-social construct, its dimensions, and applications. Journal of Vocational Behavior, 65(1), 14-38.

Grandey, A. A., Cordeiro, B. L. y Crouter, A. C. (2005). A longitudinal and multisource test of the work-family conflict and job satisfaction. Journal of Occupational and Organizational Psychology, 78(3), 305-323.

Guthrie, J. P. (2001). High involvement work practices, turnover, and productivity: Evidence from New Zealand. Academy of Management Journal, 44(1), 180-190.

Hackman, J. R. y Lawler, E. E. III (1971). Employee reaction to job characteristics. Journal of Applied Psychology, 55(3), 259-286.

Hackman J. R. y Oldham, G. R. (1976). Motivation through design of work. Organizational behaviour and human performance, 16(2), 250-279.

Hackman, J. R. y Oldham, G. R. (1980). Work redesign. Reading, MA: Addison-Wesley

Hamel, G. y Prahalad, C. K. (1995). Compitiendo por el futuro. Barcelona: Ariel.

Herrera, J. (2001). Dirección de recursos humanos. Un enfoque de administración de empresas. Valencia: ACDE ediciones.

Herzberg, F. (1966). Work and the nature of man. Cleveland, Ohio: The World Publishing Company.

Herzberg, F. (1968). One more time: How do you motivate employees? Harvard Business Review, 46(1), 53-62.

Higgins, C. A., Duxbury, L. E. e Irving, R. H. (1992). Work-Family Conflict in the Dual-Career Family. Organizational Behavior and Human Decision Processes, 51(1), 51-75.

Hillage, J. y Pollard, E. (1998). Employability: developing a framework for policy analysis. Londres: Department for Education and Employment.

Hom, P., Caranikas-Walker, F., Prussia, G. y Griffeth, R. A. (1992). A meta-analytical structural equations analysis of a model of employee turnover. Journal of Applied Psychology, 77(6), 890-909.

Huselid, M. A. (1995). The impact of human resource management practices on turnover, productivity, and corporate financial performance. Academy of Management Journal, 38(3), 635-672.

Ichniowski, C., Shaw, K., y Prennushi, G. (1997). The effects of human resource management practices on productivity: A study of steel finishing lines. The American Economic Review, 87(3), 291-313.

Isaac, R. G.; Zerbe, W. J. y Pitt, D. C. (2001). Leadership and motivation: The effective application of expectancy theory. Journal of Managerial Issues, 13(2), 212-226.

Jensen, M. (1994). Self-interest, altruism, incentives and agency theory. Journal of Applied Corporate Finance, 7(2), 40-45.

Jex, S. M. y Gudanowski, D. M. (1992). Efficacy beliefs and work stress: An exploratory study. Journal of Organizational Behavior, 13(5), 509-517.

Kamoche, K. (1996). Strategic human resource management within a resource-capability view of the firm. Journal of Management Studies, 33(2), 213-33. 
Kopleman, R. E. y Thompson, P. H. (1976). Boundary conditions for expectancy theory predictions of work motivation and job performance. Academy of Management Journal, 19(2), 237-258.

Korn Ferry International (2003). 62\% of Global Executives dissatisfied with current positions. Recuperado de http://www.kornferry.com/Library/ Process.asp?P=PR_Detail\&CID=491\&LID=1

Krugman, P. y Venables, A. (1990). Integration and the competitiveness of peripherical industry. En C. Bliss y J. Braga (Eds.), Unity with Diversity within the European Economy: The Community's southern Frontier. Cambridge University Press.

Lado, A. A. y Wilson, M. C. (1994). Human resource systems and sustained competitive advantage: A competency-based perspective. Academy of Management Review, 19(4), 699-727.

Latham, G. y Locke, E. (2002). Building a practically useful theory of goal setting and task motivation. American Psychologist, 9(57), 705-17.

Latham, G. y Yukl, G. A. (1975). A review of research on the application of goal setting in organizations. Academy of Management Journal, 18, 824-845.

Lee, T. W. (1988). How job satisfaction leads to employee turnover. Journal of Business and Psychology, 2(3), 263-271.

Lee, C., Ashford, S. J. y Bobko, P. (1990). Interactive effects of type A behaviour, and perceived control on worker performance, job satisfaction, and somatic complaints. Academy of Management Journal, 33(4), 870-881.

Lepak, D. y Snell, S. (1999). The human resource architecture: Toward a theory of human capital allocation and development. Academy of Management Review, 24(1), 31-48.

Lepak, D. P. y Snell, S. A. (2002). Examining the human resource architecture: The relationship among human capital, employment and human resource configurations. Journal of Management, 28(4), 517-543.

Locke E. A. (1968). Toward a theory of task motivation and incentives. Organizational behavior and human performance, 3(2), 157-189.

Locke, E. A., Shaw, K. N., Saari, L. M. y Latham, G. P. (1981). Goal setting and task performance: 19691980. Psychological Bulletin, 90(1), 125-52.

Lourel, M., Ford, M. T., Gamassou, C. E., Gueguen, N. y Hartmann, A. (2009). Negative and positive spillover between work and home. relationships to perceived stress of job satisfaction. Journal of Managerial Psychology, 24(5), 438-449.

Lum, L., Kervin, J., Clark, K., Reid, F. y Sirola, W. (1998). Explaining nursing turnover intent: Job satisfaction, pay satisfaction or organizational commitment? Journal of Organizational Behavior, 19, 305-320.
MacDuffie, J. P. (1995). Human resource bundles and manufacturing performance: Organizational logic and flexible production systems in the world auto industry. Industrial and Labor Relations Review, 48(2), 197-221.

Maier, N. R. F. (1963). Psicología industrial. Madrid: Rialp.

Mangione, T. W. y Quinn, R. P. (1975). Job satisfaction, counterproductive behavior and drug use at work. Journal of Applied Psychology, 60(1), 114-116.

Martín-Alcázar, F., Romero-Fernández, P. M. y Sánchez-Gardey, G. (2005). Strategic human resource management: integrating the universalistic, contingent, configurational and contextual perspectives. International Journal of Human Resource Management, 16(5), 633-659.

Maslow, A. (1943). A theory of human motivation. Psychological Review, 50, 370-396.

Maslow, A. (1954). Motivation and personality. Nueva York: Harper \& Row.

Mathieu, J. E. (1991). A cross-level nonrecursive model of the antecedents of organizational commitment and satisfaction. Journal of Applied Psychology, 76(5), 607-618.

Mathieu, J. E. y Zajac, D. M. (1990). A Review and meta-analysis of the antecedents, correlates and consequences of organizational commitment. Psychological Bulleting, 108(2), 171-194.

McClelland, D. C. (1951). Personality. Nueva York: Dryden Press.

McClelland, D. C. (1965). Achievement motivation can be developed. Harvard Business Review, 43(6), 6-24.

McEvoy, G. M. y Cascio, W. F. (1985). Strategies for reducing employee turnover: A meta-analysis. Journal of Applied Psychology, 70(2), 342-353.

McNeely, B. L. y Meglino, B. M. (1994). The role of dispositional and situational antecedents in prosocial organizational behaviour: An examination of the intended beneficiaries of prosocial behaviour. Journal of Applied Psychology, 79(6), 836-844.

Miles, R. E. y Snow, Ch. C. (1984): Designing strategic human resource systems. Organizational Dynamics, 13(1), 36-52.

Motowidlo, S. J. (1984). Does job satisfaction lead to consideration and personal sensitivity?. Academy of Management Journal, 27(4), 910-915.

Mouhoud, E. (1989). Les stratégies de relocalisation des firmes multinationales. Revue d'Economie Politique, 99(1), 96-122.

Organización Internacional del Trabajo-orT. (2001). La incidencia en el empleo de las fusiones y adquisiciones en el sector de los servicios financieros y de la banca. Ginebra: oIT, Programa de Actividades Sectoriales.

Organización Internacional del Trabajo-orT. (2003). La incidencia en el empleo de las fusiones y 
adquisiciones en el sector del comercio. Ginebra: oIT, Programa de Actividades Sectoriales.

Organ, D. y Ryan, K. A. (1995). A meta-analytic review of attitudinal and dispositional predictors of organizational behavior. Personnel Psychology, 48(4), 775-802.

Perlow, L. A. (1997). Finding time. Ithaca, Nueva York: Cornell University Press.

Pfeffer, J. (1998). Seven practices of successful organization. California Management Review, 40(2). 96-124.

Pfeffer, J. (2007). Human resources from an organizational behaviour perspective: Some paradoxes explained. Journal of Economic Perspectives, 21(4), 115-134.

Porter, L. y Lawler, E. E. (1968). Managerial attitudes and performance. Homewood: Richard D. Irwin Inc., Dorsey Press.

Rafaeli, A. (1989). When cashiers meet customers: An Analysis of the Role of Supermarket Cashiers. Academy of Journal Management, 32(2), 245-273.

Rogers, J. D., Clow, K. E. y Kash, T. J. (1994). Increasing job satisfaction of service personnel. Journal of Services Marketing, 8(1), 14-26.

Saari, L. M. y Judge, T. A. (2004). Employee attitudes and job satisfaction. Human Resource Management, 43(2), 305-407.

Schneider, B. y Bowen, D. E. (1985). Employee and customer perceptions of service in banks: Replication and extension. Journal of Applied Psychology, $70(3), 423-433$.

Schuler, R. S. (1987). Personnel and human resource management choices and organizational strategy. Human Resource Planning, 10(1), 1-17.

Schuler, R. S. y Jackson, S. E. (1987). Linking competitive strategies with human resource management. Academy of Management Executive, 1(3), 207-219.

Shirom, A. (1989). Burn out in work organizations. En C. L. Cooper e I. T. Robertson (Eds.), International review of industrial and organizational psychology (pp. 25-48). Chichester, RU: John Wiley \& Sons.

Shore, L. M., Newton, L. A. y Thornton III, G. C. (1990). Job and organizational attitudes in relation to employee behavioural intentions. Journal of Organizational Behavior, 11(1), 57-67.

Silla, I. (2007). Trabajo temporal, inseguridad laboral percibida y sus implicaciones. Factores psicosociales intervinientes (Tesis doctoral). Universidad de Valencia, Valencia, España.

Skinner, B. F. (1953). Science and human behavior. Nueva York: Macmillan.

Skinner, B. F. y Fester, C. B. (1957). Schedules of reinforcement. Nueva York: Appleton-Century-Crofts.

Skinner, B. F. y Holland, J. G. (1970). The analysis of behavior: A program for self-instruction. Nueva York: McGraw Hill.
Skinner, B. F. y Rogers C. R. (1956). Some issues concerning the control of human behaviour: A symposium. Science, 124, 1057-1066.

Smith, P. C., Kendall, L. M. y Hullin, C. L. (1969). The measurement of satisfaction in work and retirement. Chicago: Rand McNally.

Snell, S. A. y Dean, J. W. (1992). Integrated manufacturing and human resource management: A human capital perspective. Academy of Management Journal, 35(3), 467-504.

Spector, P. E., Dwyer, D. J. y Jex, S. M. (1988). Relation of job stressors to affective, health and performance outcomes: A comparison of multiple data sources. Journal of Applied Psychology, 73(1), 11-19.

Tett, R. P. y Meyer, J. P. (1993). Job satisfaction, organizational commitment, turnover intention, and turnover: Path analysis based on meta-analytic findings. Personnel Psychology, 46(2), 259-293.

Tziner, A. y Birati, A. (1996). Assessing employee turnover costs: A revised approach. Human Resource Management Review, 6(2), 113-122.

Tziner, A., Kopelman, R. E. y Neomi, L. (1993). Effects of performance appraisal format on perceived goal characteristics, appraisal process satisfaction, and changes in rated job performance: A field experiment. The Journal of Psychology, 127(3), 281-292.

Ulrich, D. y Lake, D. (1991). Organizational capability. Creating competitive advantage. Academy of Management Executive, 5(1), 77-92.

Vroom, V. H. (1964). Work and motivation. Nueva York: John Wiley \& Sons.

Way, S. A. (2002). High performance work systems and intermediate indicators of firm performance within the US small business sector. Journal Management, 28(6), 765-785.

Weinert, A. B. (1985). Manual de psicología de la organización. Barcelona: Herder.

Weiss, D. J., Dawis, R. V., England, G., y Lofquist, L. (1977). Manual for the Minnesota Satisfaction Questionnaire: Minnesota Studies in Vocational Rehabilitation. Minneapolis: University of Minnesota.

Wilthagen, T. y Tros, F. (2004). The concept of 'flexicurity': a new approach to regulating employment and labour markets. Transfer, 10(2), 166-186.

World Bank Group. (2018). Inversión extranjera directa, entrada neta de capital (balanza de pagos, us\$ a precios actuales). Bancomundial.org. Recuperado de https://datos.bancomundial.org/indicador/ BX.KLT.DINV.CD.WD? name_desc=false

Wright, P. M., Dunford, B. B. y Snell, S. A. (2001). Human resources and the resource based view of the firm. Journal of Management, 27(6), 701-21. 